\title{
Dur Labeur Des Femmes Bayam-Sellam Dans L'approvisionnement Des Marchés De La Ville De Douala (Cameroun) En Banane Plantain
}

\author{
Chadji Rosine (Ingénieur des Travaux Agricoles) \\ Institut d'Agriculture et de Gestion d'Obala (ISAGO), Obala, Cameroun \\ Folefack Denis Pompidou (Maitre de Recherche, PhD) \\ Centre Africain de Recherches sur Bananiers et Plantains (CARBAP), \\ Unité de Recherche, Système d'information économique et mesure de \\ l'impact des innovations (SIEMI), Njombé, Cameroun
}

Doi:10.19044/esj.2018.v14n32p115 URL:http://dx.doi.org/10.19044/esj.2018.v14n32p115

\begin{abstract}
The main aim of this study is to evaluate the working conditions of some women who supply plantain bananas to markets in Douala. Surveys were conducted among 60 women along National Road 5. This study revealed that the supply chain is dominated by older women ( 47 years old). Nearly half were married contrary to what one might have thought. Furthermore majority of these women were from the Western Region (76\%) and were more experience; to carry out this activity Nevertheless they were faced with difficulties such as Long working hours from 4 am to midnight (than 15 hours) s, braving the risk of aggression and accidents. Their goods were best transported by bus or trucks to the rural markets. In order to purchase this product they are subjected to a lot of challenges and in addition to this, their physical condition must be in order. Another challenge faced by these women is the long waiting hours for trucks or buses to come by. This can take 2-6 hours before the buses arrive. In addition, the activity is mostly appreciated by the actors who do contribute to the economic empowerment of women, because it is profitable. However, they are faced with several constraints such as: theft, insecurity; high cost of transportation; lack of information; poor road infrastructure; police harassment; difficulties to have a warehouse to facilitate the storage and conservation conditions, etc. Due to these constraints, the commercial potential of women is hindered and much lower than it could be.
\end{abstract}

Keywords: Women, supply, bayam-sellam, plantain banana, markets, Douala. 


\section{Résumé}

L'étude vise a évaluer les conditions de travail des femmes qui approvisionnent des marchés de la ville de Douala en banane plantain, ainsi que la rentabilité de ce commerce. Des enquêtes ont été menées auprès de 60 femmes le long de la route nationale $n^{\circ} 5$. L'étude montre que la chaine d'approvisionnement est dominée par les femmes âgées. Près de la moitié sont mariés contrairement à ce qu'on pourrait penser que l'activité est réservée aux femmes célibataires. Elles sont majoritairement $(76 \%)$ originaires de la région de l'Ouest et très expérimentées dans le commerce. Des longues journées de travail de plus de $15 \mathrm{~h}$, allant de $4 \mathrm{~h}$ du matin jusqu'à très tard entre 21 et $24 \mathrm{~h}$, bravant des risques d'agression et d'accidents. Le mode transport couramment utilisé est les bus ou cars vers les marchés ruraux et les camions au retour. L'achat des produits est soumis à une forte compétition et il faut se doter d'une bonne condition physique. Un fait marquant et stressant pour les femmes, c'est le délai d'attente du camion qui est très long ( 2 à 6 heures) au bord des routes. En outre, l'activité est diversement appréciée par les acteurs et contribue tout de même à l'autonomisation économique des femmes, car, elle est rentable. Cependant, elles font face à de fortes contraintes : vols et insécurité ; cherté des coûts de transport; manque d'information; tracasseries routières et policières; difficultés de stockage et conservation, etc. En raison des contraintes, le potentiel commercial des femmes est entravé et bien inférieur à ce qu'elle pourrait être.

Mots clés : Femmes, approvisionnement, bayam-sellam, banane plantain, marchés, Douala.

\section{Introduction}

Le bananier plantain est l'une des cultures vivrières les plus importantes au Cameroun. Son rôle est essentiel dans l'alimentation quotidienne, économiquement de nombreux petits producteurs en dépendent et la banane plantain occupe la troisième place dans l'échelle des féculents après le riz et le manioc (Houngbo, 2014). L'essor du marché du plantain au Cameroun se structure principalement autour de deux pôles urbains à savoir: Douala, capitale économique ayant le port principal et Yaoundé la capitale politique. Ces deux pôles de consommation sont relativement indépendants l'un de l'autre dans leurs zones d'approvisionnement. Le commerce du plantain emploie environ 38000 personnes, les revenus obtenus servent principalement aux dépenses de santé et de scolarisation (Bikoï et al., 2012; Fongang et al., 2016).

La demande de la banane plantain est en pleine croissance à Douala suite à la pression démographique, l'accroissement de la population urbaine et du fait de ses diverses formes de consommation de la banane plantain : mûres, 
cuites, séchées ou frites, transformées en farine, etc. (Bikoï et al., 2004 ; Tomekpe, 2006 ; Bikoï et al., 2012 ; Wandji, 2015 ; Pane, 2015). Les marchés de la ville de Douala sont approvisionnés majoritairement en banane plantain provenant des bassins de productions des régions du Littoral, Sud-Ouest et Ouest par les femmes Bayam-Sellam, dont leurs proportions respectives sont : détaillants (86\%), collecteurs (74\%), grossistes (64\%) (Folefack et al., 2017).

Dans un contexte de crise alimentaire globalisée où la «question de l'approvisionnement des villes africaines en produits agroalimentaires demeure un enjeu majeur » (Fouda, 2009), cette activité, née de la nécessité d'approvisionner les villes en ressources alimentaires, est capitale. Cependant, le travail de ces amazones de commerce est miné par de fortes contraintes auxquelles les femmes doivent faire face, notamment : le faible accès au crédit bancaire; l'illettrisme qui engendre une méconnaissance des règles commerciales en vigueur; la faible organisation des femmes ; l'accès difficile dans les marchés ruraux; les conditions de travail assez difficiles, etc. (Mohamadou, 2016; Aboudou et al., 2017).

Une récente étude menée par Folefack et al (2017) a permis de constater que malgré le caractère rentable de l'activité des femmes, les conditions de travail rudes occupent une place de choix dans l'approvisionnement des marchés en banane plantain. Face ces conditions difficiles, ces dernières ne disposent très peu d'alternatives et d'opportunités et sont contraintes parfois à rester dans l'activité. Pour une dynamisation de l'activité, le défi que doivent relever les acteurs de la filière banane plantain est d'améliorer les conditions de travail des femmes, afin d'attirer davantage de jeunes filles en chômage dans l'activité. Cependant, très peu d'informations sont disponibles pour évaluer les conditions réelles de travail de ces braves dames, d'où l'importance et l'originalité de cette étude. Ce travail vise à évaluer les conditions de travail des femmes qui approvisionnent les marchés de la ville de Douala en banane plantain ainsi que la rentabilité de ce commerce.

\section{Méthodologie}

\section{Cadre de l'étude}

L'étude a été réalisée au Cameroun, dans la région du Littoral et principalement sur la route nationale $n^{\circ} 5$ (axe Douala - Bafoussam). Elle s'inscrit dans la logique dans la continuité d'une étude réalisée dans le cadre du projet IRAD-C2D/PAR Plantain en 2016 portant sur l'analyse de la performance du système d'approvisionnement de la ville de Douala en banane plantain. Cette étude a permis de montrer le rôle déterminant des femmes dans le commerce de la banane plantain, et elles évoquaient fréquemment les difficiles conditions de travail. Dans le cadre cette étude, le flux de littoral retient notre attention (flux en vert sur la figure 1). 


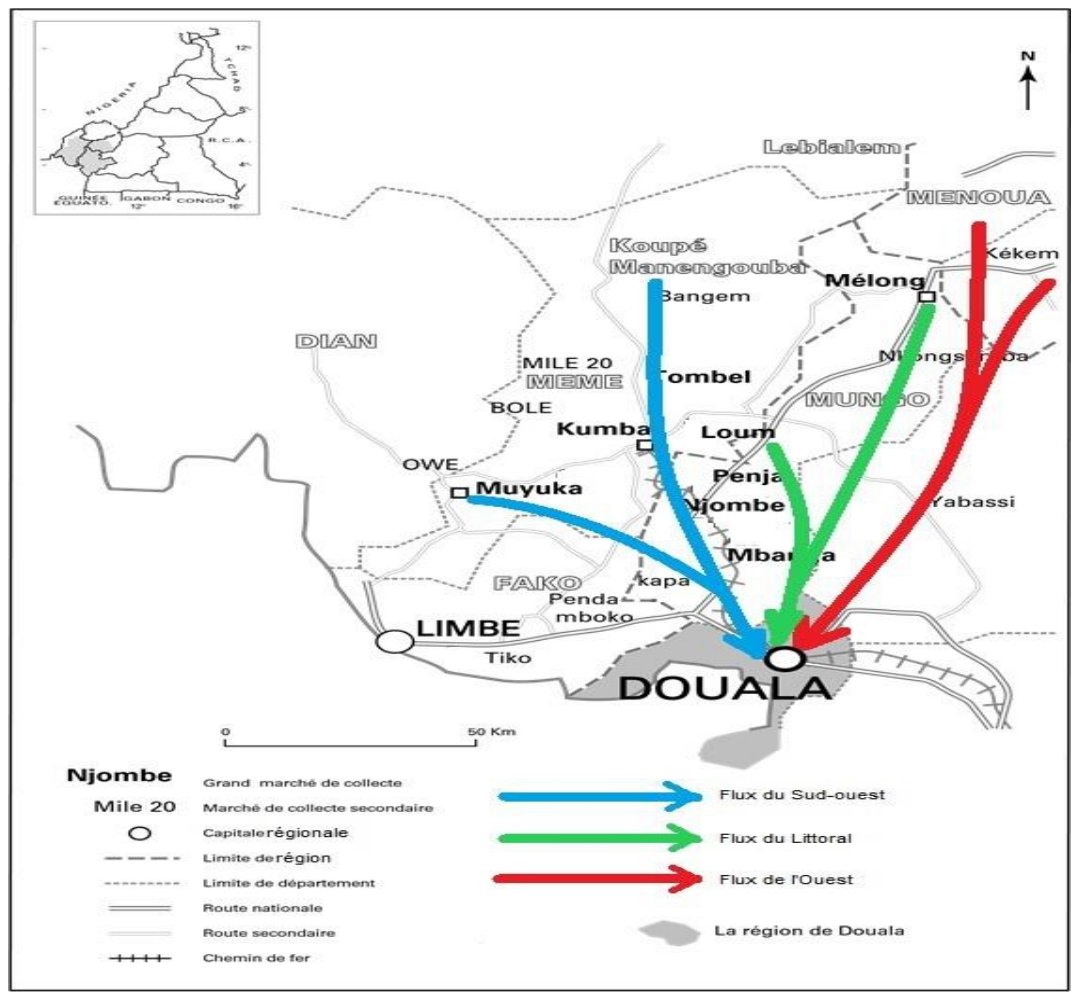

Source : Mohamadou, 2016

Figure 1 : Flux d'approvisionnement de la ville Douala en banane plantain

\section{Méthodes d'enquête}

Les données ont été collectées à travers des enquêtes auprès des femmes, plus précisément celles qui s'activent dans l'approvisionnement des marchés de la ville de Douala en banane plantain à partir des marchés situés aux abords de la route nationale $n^{\circ} 5$. Ainsi, 60 femmes Buyam-Sellam choisies au hasard ont été enquêtées par le biais de questionnaires structurés dans les marchés des localités de : Loum, Njombé, Melong, Mbanga, Penja et Souza. La structure du questionnaire était focalisée sur: caractéristiques socioéconomiques; produits achetés et les stratégies; difficiles conditions de travail; perception des acteurs; risques et contraintes; options stratégies. Les observations directes ont été faites en parallèle avec la collecte des données, ainsi qu'une analyse documentaire.

\section{Traitement statistique}

Enfin, les données collectées ont été saisies dans le logiciel Microsoft Excel et analysées dans SPSS 20. Des statistiques descriptives (fréquence, moyenne, écart type) ont été effectuées pour obtenir des résultats que nous présentons par la suite. 


\section{Résultats et discussion \\ Cadrage et identification des acteurs}

Au Cameroun, les femmes exerçant l'activité d'approvisionnement des marchés en produits agricoles sont appelées communément « Bayam-Sellam », un mot tiré du pidgin local qui signifie « acheter et vendre ». Leur activité est d'acheter des produits agricoles dans les marchés ruraux pour les revendre en gros ou au détail dans les villes. Selon Ngo (2011), les femmes Bayam-Sellam peuvent être classées en trois catégories qui travaillent dans une logique de chaîne et de filière d'approvisionnement "périphérie-centre » et «centrecentre ». La logique catégorielle est la suivante: les Bayam-Sellam productrices de villages, les Bayam-Sellam relais villages-villes, et les BayamSellam détaillantes fixes des villes. Les premières, encore appelées Bayam de proximité, sont présentes quotidiennement dans leur ménage et continuent d'assumer en parallèle les autres responsabilités traditionnelles des femmes. Les deuxièmes sont celles qui habitent la ville, mais se déplacent vers les marchés périodiques des petites villes selon un calendrier précis, en fonction des besoins d'achat et de vente. Quant aux troisièmes, qui habitent continûment en ville, elles s'approvisionnent chez les Bayam-Sellam relais et vendent au marché urbain, soit sur les comptoirs aménagés par les communes qu'elles louent, soit dans des espaces « sauvages » où elles étalent leurs marchandises. C'est dans cette catégorie que l'on trouve des Bayam-Sellam beaucoup plus scolarisées. Dans le cadre de cette étude, c'est la deuxième catégorie qui retient notre attention.

Dans la région du littoral, les jeunes femmes ne semblent pas être intéressées par cette activité, car l'âge moyen de ces commerçantes est de 47 ans, montrant qu'elles sont vieillissantes. Les jeunes femmes sont confrontées à des pressions sociales et familiales qui les empêchent d'exercer l'activité, alors que c'est un vivier d'emploi qui peut contribuer à la lutte contre la pauvreté et le chômage.

Les femmes ayant le niveau primaire et secondaire sont les plus représentées, avec respectivement 25 et $31 \%$. Le fait marquant c'est qu'aucune femme n'a le niveau supérieur. Le statut dominant est celui des mariés qui compte près de $53 \%$, contrairement à ce qui se dit que cette activité serait réservée plus aux femmes célibataires. Ensuite viennent les célibataires et les veuves avec $18 \%$ et enfin, les divorcés sont les moins représentés (10 \%). La proportion des femmes vivant seules est quand même importante et celles-là n'ont certainement pas de contraintes des époux.

Les femmes rencontrées sont pour la plupart $(76 \%)$ originaires de la région de l'ouest du Cameroun. Elles ont une expérience assez longue dans leur domaine d'activité. Le nombre de personnes à charge moyen est de 4 , cela est un catalyseur des femmes dans cette activité. Le nombre d'années d'expérience moyen est de 12 ans. Le nombre d'années assez important dans 
l'exercice de cette activité traduirait le fait que les acteurs sont stables dans cette activité. Quand ils commencent, ils n'abandonnent pas facilement, du fait de la rentabilité certaine et du manque d'opportunités alternatives.

Enfin, l'étude montre que l'organisation des acteurs en associations formelles est assez faible et il n'existe pas d'interprofession spécifique des acteurs de la filière banane plantain pour la défense des intérêts des acteurs. Il faut tout de même signaler qu'il existe l'Association des Bayam-Selam du Cameroun (ASBY) créée en 2004. Elle a mis sur pied un concept qui vise à fédérer tous les acteurs intervenant dans les circuits de production et de commercialisation des biens agricoles, pour l'accroissement de la production et mise sur pied d'une chaîne de commercialisation. Cependant, les femmes enquêtées affirment ne pas être membres de cette association fédérale. En effet, cette association élitiste et regroupant plus les femmes dans les grands marchés des grandes villes. En plus, on note aussi, la réticence des femmes aux regroupements dans les marchés, elles évoquent de mauvais souvenirs des regroupements antérieurs, notamment, les cas de sorcellerie dans les marchés, le problème de gouvernance, qui ont conduit à la dislocation des groupes.

\section{Difficiles conditions de travail des femmes Longues journées de travail}

Les journées de travail des femmes exerçant l'activité d'achat et vente des produits agricoles sont très longues et denses. La figure 2 nous donne des informations sur les heures de départ et de retour à la maison.

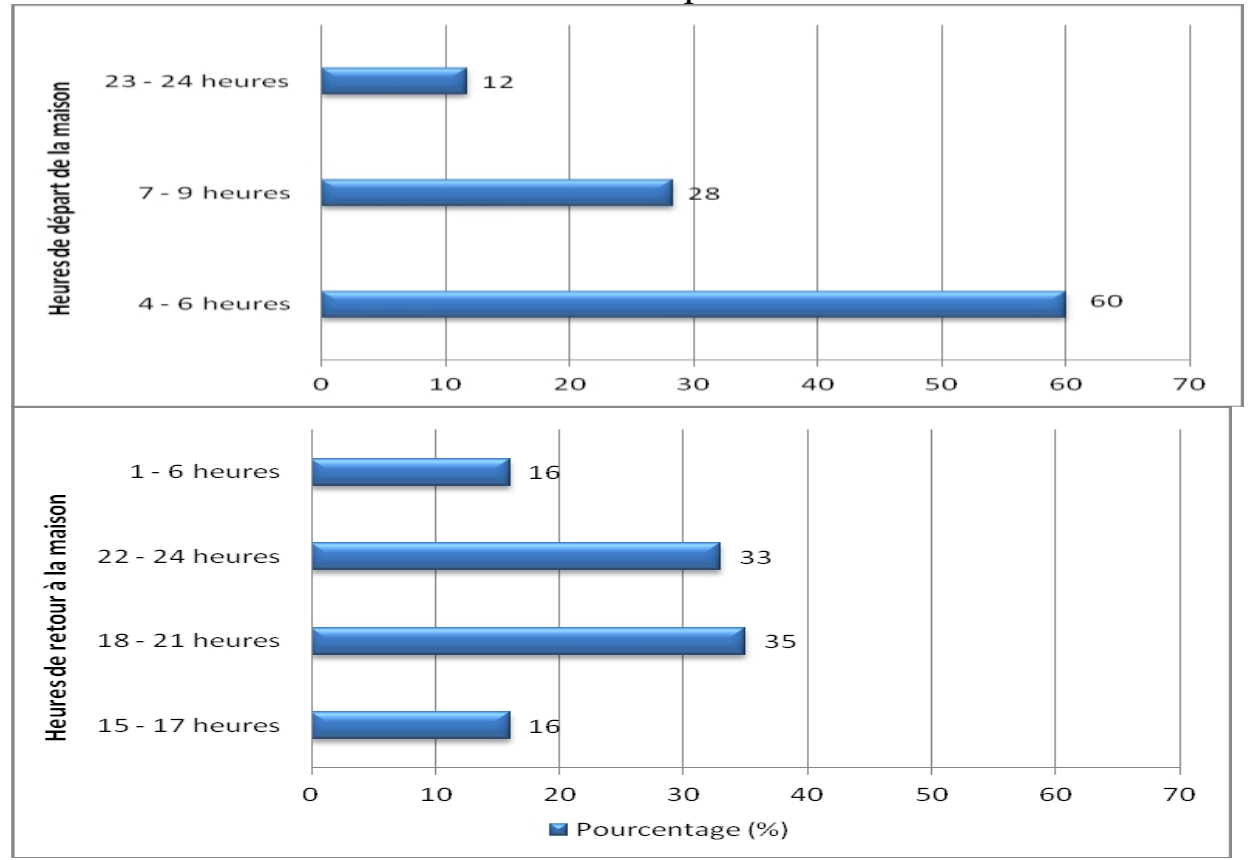

Figure 2 : Heures départ et retour de la maison 
Dans leurs activités, les femmes se lèvent aux premiers chants du coq (à partir de 4 heures du matin), pour se rendre dans des marchés ruraux. Elles parcourent des kilomètres au quotidien pour acheter des aliments qu'elles vendent par la suite dans les marchés camerounais. Notre étude montre que près de $60 \%$ des femmes commerçantes quittent leurs maisons entre 4 et 6 heures du matin selon la distance, bravant des risques d'agression et d'accidents pour se rendre à leurs lieux de travail (marchés d'approvisionnement). Lorsque les marchés sont proches de la ville de Douala, elles peuvent quitter après le lever du jour entre 7 et 9 heures pour s'y rendre, tandis que d'autres ( $12 \%$ ) voyagent la veille du marché entre 22 et 24 heures pour aller dormir dans les marchés, afin de commencer les achats très tôt au lever du jour.

Pour ce qui est des heures de retour à la maison, elles dépendent fortement de la disponibilité d'un moyen de transport pour l'acheminement des marchandises achetées. Plus, le délai d'attente de la voiture est court, plus tôt elles arrivent au marché de Douala pour stocker les marchandises en attendant la vente le lendemain et retourner dans les domiciles. Très peu retourne avant la nuit, environ $16 \%$. Une proportion plus importante des femmes retourne entre 18 et 21 heures et 22 et 24 heures. Lorsqu'elles n'ont pas la chance de trouver un moyen de transport, elles arrivent dans les marchés à Douala et à la maison à des heures très tardives et peuvent être obligées de passer la nuit dans les marchés et écouler leurs marchandises au lever du jour, avant de retourner à la maison. À partir de ces résultats, nous constatons des journées de travail des femmes très longues (plus de 15 heures). Au Cameroun selon la Loi no 92/007 du 14 août 1992, portant code du travail, dans tous les établissements publics ou privés non agricoles, la durée de travail ne peut excéder quarante (40) heures par semaine, soit 8 heures par jour. Le commerce des produits agricoles par les femmes fait partie des activités les plus pénibles.

\section{Moyens de transport utilisés et délai d'attente de véhicule}

Plusieurs moyens sont utilisés pour le transport des marchandises des marchés ruraux vers la ville de Douala.

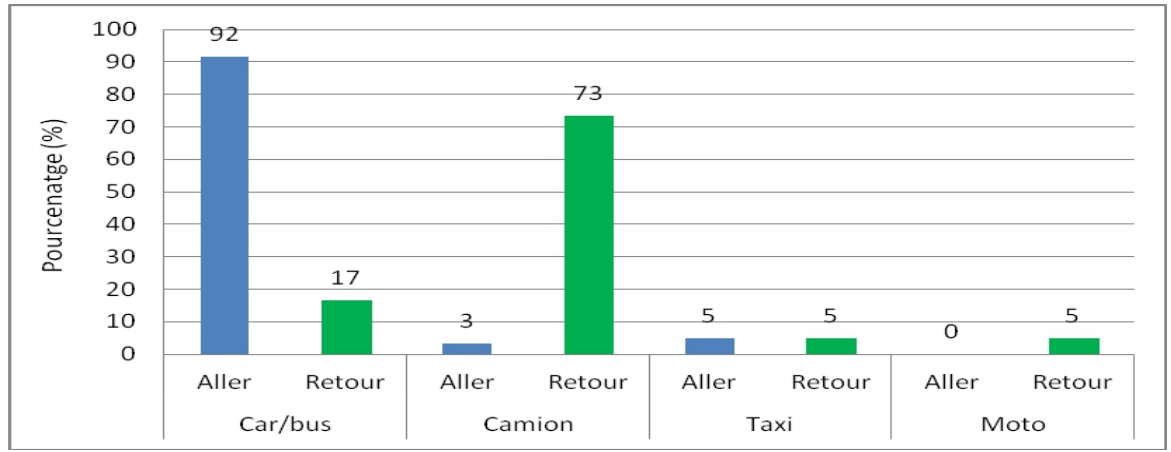

Figure 3 : Moyens de transport utilisés 
Le mode transport dépend de la destination et de la proximité ou pas du marché. Selon des femmes, pour se rendre dans les marchés ruraux, depuis la ville de Douala, elles utilisent dans une large majorité (92\%) les cars ou les bus. Dans ce sens du voyage, elles voyagent relativement dans de bonnes conditions, comme la plupart des marchés sont situés près de la route nationale. Les autres moyens de transport sont utilisés de façon très marginale.

En possession de leurs produits, les Bayam-Sellam utilisent plusieurs modes de transport afin de rejoindre les différents points de vente dans la ville de Douala. Ainsi, dans leurs parcours de combattant avec des quantités importantes de marchandises achetées et encombrantes, les camions sont les moyens de transport le plus utilisé par près de $73 \%$ des femmes pour acheminer les produits agricoles vers la ville de Douala. Les camions sont plus utilisés à cause de leur grande capacité de transport de produits agricoles achetés dans les marchés y compris la banane plantain. Par ailleurs, il faut noter que lors du voyage retour les cars sont utilisés surtout lorsque les femmes n'ont pas pu acheter des grandes quantités de produits dans un marché. Enfin, il faut aussi signaler l'utilisation des taxis de ville alors que les motos sont utilisées de façon marginale particulièrement lorsque la distance est courte entre les marchés ruraux et ceux de Douala. Le cout du transport représente entre 5 et $7 \%$ du prix de vente des produits dans les marchés de Douala.

L'étude révèle que la problématique du transport des produits achetés dans les marchés ruraux vers la ville de Douala est très complexe. En effet, les femmes éprouvent d'énormes difficultés pour trouver un camion pour le transport des marchandises. Selon ces femmes, le délai d'attente d'une hypothétique voiture est très long pouvant aller de 1 à 4 heures au bord des routes. Dans le long de la route nationale $\mathrm{n}^{\circ} 5$, il est très fréquent de rencontrer des femmes assises en bordure des routes dans la nuit vers 21 et 22 heures à la recherche d'une voiture pour transport des produits achetés. Dans les cas extrêmes, certaines femmes affirment souvent passer la nuit en bordure de la route à la recherche de la voiture. Dans ces cas, elles font face à des risques de vols et de pertes des marchandises dans les marchés ruraux.

\section{Absence d'infrastructures de marchés}

Les marchés constituent des lieux de rencontre privilégiés entre les producteurs et commerçants. Si dans les plus grandes villes et les cités de moindre importance, les ventes ont lieu tous les jours, les quantités de plantains qui arrivent sur les étalages varient selon un mouvement assez régulier des acheteurs. Dans les marchés ruraux, les marchés ont lieu généralement une fois par semaine.

Dans les marchés ruraux, nous observons une absence d'infrastructures d'accueil pour les acteurs tels que les hangars, les magasins. En plus, il faut remarquer que l'accès à ces marchés depuis la route nationale 5 est très difficile 
du fait du mauvais état des routes rurales, surtout en saison des pluies. Aussi, du fait que certains marchés débutent très tôt en matinée, les vendeurs et les acheteurs sont parfois obligés de passer les nuits sur place à la belle étoile avec tous les risques associés.

\section{Perception de l'activité}

L'approvisionnement des marchés de la ville de Douala est diversement apprécié par les acteurs qui le réalisent. Selon les femmes exerçant cette activité, malgré le fait que l'activité est rentable et permet de nourrir la famille et subvenir aux besoins, le travail est très difficile. La plupart estiment qu'elles souffrent beaucoup pour contribuer à nourrir les villes camerounaises. Pour certaines femmes avec cette activité, elles perdent leur beauté au fil des années, et que plusieurs personnes les regardent avec beaucoup de mépris et de pitié dans les marchés. Certains avouent même rester dans l'activité parce qu'elles n'ont pas de choix, et qu'elles ne peuvent pas accepter leurs propres filles exercer la même activité, dite "activité de souffrance ». Malgré cette perception pessimiste, une étude conduite par Ella (2016), montre que l'activité des Bayam-Sellam assure l'épanouissement de la femme. Cette activité rémunératrice libère la femme de nombreuses contraintes et améliore sa dignité humaine. Elle s'éloigne aussi de la mendicité et de l'oisiveté. Elle n'est plus considérée comme une simple consommatrice. Il en résulte une baisse de la prostitution, de la consommation de la drogue et de la criminalité. À travers cette activité génératrice de revenus, la femme acquiert une autonomie financière, un pouvoir économique et de nouvelles responsabilités sociales. Ceci conduit à une transformation notable des relations dans les ménages et dans la société.

Par ailleurs dans l'entourage immédiat, les époux acceptent malgré eux que leurs femmes exercent cette activité. Avec la vie chère, les hommes n'ont pas de choix de laisser leurs femmes exercer cette activité pour contribuer aux charges familiales. Cependant, avec les exigences de cette l'activité, qui font que les femmes sont toujours en voyage et dans les marchés, elles ne sont plus disponibles pour s'occuper de leur mari et de la famille. Dans ce contexte, c'est les enfants surtout la fille ainée qui prend le relais. Les époux ne sont pas toujours d'accord dans cet état de chose, ce qui créait des tensions tous les temps au sein des couples, en plus certains ont des soupçons d'infidélité de leurs femmes.

Par ailleurs, certaines femmes sont souvent insultées parce que, à peine rentrées des villages où elles achètent leurs marchandises, elles ne se lavent pas, car elles sont pressées de livrer à leurs clientes. Leur accoutrement dans les marchés n'est pas attirant. Cela semble être un facteur défavorable pour attirer les jeunes filles en chômage dans cette activité. Les jeunes femmes estiment que l'activité est très difficile et qu'elles n'ont pas de force physique 
pour parcourir les marchés et acheter les produits. En plus, elles ont encore des enfants très petits, il faut du temps pour s'occuper d'eux. Sans toutefois oubliée, le fait que les époux refusent à leur jeune femme d'exercer l'activité. En somme, les conditions de travail ne sont pas décentes et ne permettent pas aux femmes un épanouissement moralement.

\section{Activité génératrice de revenus pour l'autonomisation des femmes}

Les principales motivations des femmes pour le choix de cette activité de commerce de la banane plantain sont entre autres : les problèmes financiers à résoudre, la rentabilité et la pauvreté. Par ailleurs, les femmes n'ayant pas accès aux banques classiques, les femmes Bayam-Sellam ont trouvé dans les tontines, systèmes de rentes collectives, un outil économique d'emprunt, d'épargne et de financement informel de leurs activités. Les tontines sont en effet l'expression d'une solidarité plurielle fondée sur les valeurs traditionnelles de solidarité, de confiance réciproque, d'entraide et de respect mutuel des principes fondant les liens interpersonnels (Fouda, 2009).

Ainsi, une analyse économique permet de se prononcer clairement sur la rentabilité évoquée par des acteurs de cette activité. Pour le faire, un compte d'exploitation mensuelle de l'activité a été réalisé avec les données collectées auprès des femmes commerçantes. De ce fait, le compte d'exploitation mensuel est réalisé à partir des données collectées. Les chiffres peuvent être résumés de la manière suivante. Le nombre de régimes manipulés par les femmes tourne autour de 500 en moyenne. Les dépenses moyennes mensuelles estimées pour les femmes à partir de la désagrégation des prix (frais d'achat du produit, de transport, de manutention, de stockage, de place au marché ...) sont évaluées à 1968760 FCFA. Les chiffres d'affaires moyens mensuels sont évalués 2366372 FCFA et des profits bruts moyens de presque 397612 FCFA. Il est de loin supérieur au salaire d'un cadre supérieur (catégorie A2) de fonction publique camerounaise. Les femmes BayamSellam brassent une masse d'argent non négligeable dans leurs activités. Selon Ella (2016), elles se retrouvent souvent au sein de nombreuses associations pour la sécurisation de leurs avoirs financiers. Ces cotisations qui constituent une épargne peuvent être quotidiennes, hebdomadaires ou mensuelles.

Cela montre que l'activité est génératrice de revenus et permet à ces femmes de se rendre autonomes. Dans les familles, l'argent si durement gagné par les femmes sert à payer la scolarité, l'hospitalisation et la nutrition de leurs enfants. Certaines paient aussi la location des maisons qu'elles occupent. 
Tableau 1 : Analyse de la rentabilité (compte d'exploitation mensuel)

\begin{tabular}{lllcc}
\hline Rubrique & Unité & Quantité & $\begin{array}{c}\text { Coût unitaire } \\
\text { (FCFA) }\end{array}$ & $\begin{array}{c}\text { Coût total } \\
\text { (FCFA) }\end{array}$ \\
\hline Dépenses & & & & \\
\hline Achat plantain & Régime & 500 & 3491 & 1744262 \\
Transport régime & Forfait & 1 & 49805 & 49805 \\
Transport dans la ville & Forfait & 1 & 14663 & 14663 \\
Frais de voyage & Billets & 5 & 5000 & 25000 \\
Ticket du marché & Forfait & 1 & 4377 & 4377 \\
Gardiennage & Mois & 1 & 1078 & 1078 \\
Frais d'occupation temporaire de & Mois & 1 & 2625 & 2625 \\
voie publique & H/J & 5 & 2412 & 12060 \\
Manutention & Mois & 1 & 1854 & 1854 \\
Location Magasin & Forfait & 1 & 1500 & 1500 \\
Balayage & Forfait & 1 & 3500 & 3500 \\
Autres dépenses & Forfait & 5 & 1857 & 9286 \\
Droits communaux & Forfait & 5 & 1000 & 5000 \\
Droit de passage & Mois & 1 & 93750 & 93750 \\
Imprévus (5 \%) & & & 1968760 \\
\hline Total dépense & & & \\
\hline Recettes & Régime & 486 & 2366372 \\
\hline Vente du plantain & & & 2366372 \\
\hline Total recette & & & $\mathbf{3 9 7 6 1 2}$ \\
\hline Profit brut & & &
\end{tabular}

Fortes contraintes des femmes dans leur activité

Les femmes dans l'activité d'approvisionnement de la ville de Douala en banane plantain rencontrent plusieurs difficultés présentées dans la figure 4.

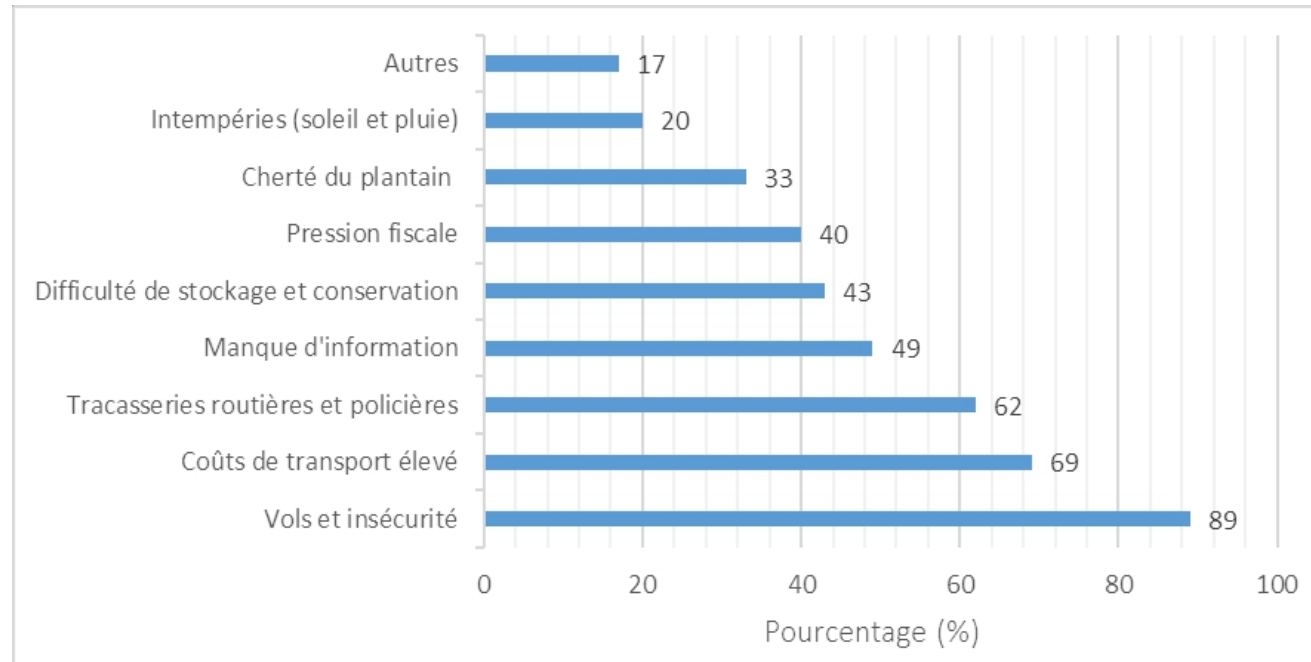

Figure 4 : Contraintes rencontrées par les femmes 
L'étude montre que le vol des plantains et l'insécurité, ainsi que les coûts élevés de transport des produits des marchés secondaires vers la ville de Douala et les diverses tracasseries routières et policières constituent selon les femmes les contraintes majeures. Ce sont des cas de vols aussi bien dans les marchés de Douala que dans les marchés ruraux où l'on rencontre des cas d'agression. Tout ceci lié au fait qu'il n'existe pas ou qu'ils sont insuffisants des structures de marchés. En effet, le manque d'infrastructure dans les marchés de la ville de Douala et les marchés ruraux font que les femmes sont très exposées au cas de vol des produits après achat. En plus, le coût élevé de transport est un facteur limitant l'activité, sans oublier la difficulté d'avoir des véhicules pour le transport des produits agricoles après l'achat dans les marchés ruraux. Ensuite, lors du transport des produits vers Douala, les transporteurs font face à de nombreuses tracasseries lors de l'acheminement des produits vers les marchés.

L'autre difficulté notoire est constituée des tracasseries routières et policières, de l'avis de presque $62 \%$ des femmes. Cet ensemble de tracasseries est constitué du nombre pléthorique de postes de contrôles routiers ainsi que les pertes de temps (souvent des heures d'immobilité) et de moyens financiers.

Le manque d'information sur les prix est aussi très contraignant selon les femmes, au Cameroun, le système d'information des marchés est inexistant, donc aucune référence fiable pour suivre l'évolution des prix dans les marchés en vue de faciliter, la prise de décision. Cette situation entraine des ruptures dans le circuit avec des moments de manque assez sévère. Ils sont jusqu'à $49 \%$ des grossistes notamment à dénoncer cette difficulté. Le pouvoir de négociation des producteurs et des consommateurs est altéré s'ils ne sont informés ni des prix pratiqués sur d'autres marchés ni du niveau des récoltes (PAM, 2013).

Le manque d'infrastructures dans les marchés entraine les difficultés de stockage et de conservation des produits évoquées par environ $43 \%$ qui pour la plupart n'ont pas des magasins (qui sont assez chers pour certains) et utilisent leurs comptoirs (qui sont souvent provisoires) comme lieux de stockage.

Les autres difficultés sont la pression fiscale à travers les taxes (impôts libératoires, droit de place dans les marchés, droit d'occupation temporaire de la voie publique, droit d'hygiène et salubrité) qui sont jugées trop élevées, constituent une contrainte assez importante. De fait, des relations conflictuelles naissent aussi entre les agents du fisc et les commerçants, situation que ces deniers trouvent stressante.

Nous notons aussi des plaintes en lien avec le prix élevé de la banane plantain dans les marchés ruraux qui rend l'approvisionnement souhaité assez difficile. La présence des commerçants étrangers dans la zone entraine une augmentation des coûts d'achat. Si le niveau de concurrence est faible, les 
commerçants peuvent plus facilement s'entendre tant sur les prix d'achat aux producteurs que sur les prix de vente aux consommateurs selon le PAM (2013); donc la hausse de concurrence pour les commerçants est assez sérieuse. Enfin, nous notons les intempéries (pluies, soleil, poussières) que ces commerçants bravent dans l'exercice de leur activité.

Toutes ces contraintes rendent l'activité assez difficile, dans ce cas, il faut s'armer de force mentale importante pour rester et continuer à exercer l'activité. La plupart des femmes commerçantes estiment qu'elles restent parce qu'elles n'ont pas d'alternatives et d'autres opportunités.

Ces fortes contraintes rencontrées par les femmes ont une similitude avec celles rencontrées par les femmes commerçantes opérant sur le corridor Abidjan-Lagos réalisé par Aboudou et al (2017), notamment : l'insuffisance des infrastructures et équipements de transport et de communication, le manque d'information des marchés et les opportunités d'affaires, pour certaines marchandises. Les femmes sont particulièrement touchées par les problèmes de tracasseries administratives au niveau des frontières du fait que la majorité d'entre elles soit analphabète et n'ait pas une bonne connaissance des dispositions en vigueur. Elles rencontrent des problèmes relatifs au transport des produits et sont aussi confrontées aux problèmes d'insécurité liée à leur personne et à leurs biens.

\section{Actions pour l'amélioration des conditions de travail des femmes}

Pour une amélioration des performances de la chaîne d'approvisionnement de la ville de Douala en banane plantain, les actions suivantes peuvent être mises en œuvre :

- Améliorer les conditions de transport de la banane plantain dans la ville de Douala. Ceci se ferait par le biais d'une baisse du prix du carburant, une diminution de contrôles routiers pour les produits vivriers périssables, un meilleur entretien d'infrastructures routières, une sensibilisation des acteurs sur le paiement des taxes formelles pour le transport des produits agricoles et les taxes informelles.

- Construire des infrastructures dans les marchés ruraux et urbains, tels que les hangars, des magasins de stockage du plantain dans les marchés.

- Promouvoir la vente de banane plantain en unité homogène (en kilogramme) (comme cela se fait dans des unités pilotes dans les centres de recherche à l'IRAD et au CARBAP, ainsi que dans d'autres pays comme la Côte d'Ivoire qui essaye actuellement de le pratiquer).

- Promouvoir le transport de la banane plantain dans les cartons comme le cas de la banane dessert, ceci pour assurer la qualité des produits et accéder facilement à la vente des produits en kilogramme dans les grandes surfaces (supermarchés). 
- Assurer une meilleure coordination tant verticale (en association ou coopérative) qu'horizontale (relations de confiance, contractuelles, de spécialisation). Cela permettrait de mieux entendre leurs doléances, de s'organiser pour la résolution de leurs problèmes (fixation et négociation de prix, le manque d'infrastructures, la circulation d'informations, l'obtention de contrats durables, les « conflits» avec les agents du fisc et des forces de l'ordre).

\section{Conclusion}

Cette étude visait à évaluer les conditions de travail des femmes qui approvisionnent des marchés de la ville de Douala en banane plantain, ainsi que la rentabilité de ce commerce. Des enquêtes ont été réalisées auprès de 60 femmes sur la route nationale $\mathrm{n}^{\circ} 5$.

Les résultats de l'étude montrent que la chaîne d'approvisionnement est nettement dominée par les femmes âgées. Les femmes ayant le niveau primaire et secondaire sont les plus représentées, avec respectivement 25 et $31 \%$. Le statut dominant est celui des mariés qui compte près de $53 \%$. Ces femmes sont pour la plupart originaires de l'ouest du Cameroun.

Les journées de travail des femmes sont très longues, elles doivent se lever très tôt entre 4 et 5 heures du matin pour se rendre dans les marchés, et elles doivent passer toute la journée dans les marchés et retourner dans leur domicile tout épuisé entre 21 et 24 heures. Le mode transport dépend de la destination des femmes, en effet, pour se rendre dans les marchés ruraux, ces femmes utilisent principalement les cars ou les bus à presque $92 \%$. Ainsi, dans le voyage retour, le camion est le moyen de transport le plus utilisé par près de $73 \%$ des femmes pour acheminer les grandes quantités des produits agricoles achetés dans les marchés de brousse.

L'activité est diversement appréciée, ainsi, selon, les femmes exerçant cette activité, le travail est très difficile et beaucoup de souffrances. Les époux acceptent malgré eux que leurs femmes exercent cette activité. Car, les exigences de cette l'activité font qu'elles ne sont plus disponibles pour s'occuper de leur mari et de la famille. C'est les enfants surtout les filles ainées qui prennent le relais. Cette activité est génératrice de revenus et contribue de façon importante à l'autonomisation des femmes. Elle est rentable et génère les profits bruts mensuels d'environ 397612 FCFA. Bien qu'elle gagne leur vie à la sueur de leur front, car, elles font face à de fortes contraintes : les vols et l'insécurité ; la cherté des coûts de transport ; le manque d'information; les tracasseries routières et policières ; les difficultés liées au stockage ; la pression fiscale et la cherté de la banane plantain.

Une amélioration des conditions de transports, avec l'adoption du transport en carton du plantain, une meilleure organisation des acteurs, une réduction des contrôles routiers et des tracasseries routières, la construction des 
infrastructures dans les marchés sont nécessaires et permettraient d'améliorer les performances du système d'approvisionnement de la ville de Douala en banane plantain.

\section{References:}

1. Aboudou, F., Oga, A., Tassoun, M. \& Alamou, K. (2017). Étude sur les problèmes spécifiques que rencontrent les femmes commerçantes sur le corridor Abidjan-Lagos. Rapport d'étude, LARES. WiLDAFAO.

2. Bikoï, A., Nkapnang, D., Ndemba, B., Ziki, E., Keleke, S. \& Ebongue, J.P. (2012). Analyse des circuits de commercialisation du plantain dans les plateformes transfrontalières Ntem, Oubangui et Congo. Planta innovation $n^{\circ}$ 91-94, juillet 2011 - juin 2012, CARBAP.

3. Bikoï, A., Nyoungou, S. \& Ngoma Moutomé, B. (2004). Analyse de la filière d'approvisionnement en plantain de la ville de Douala. Douala. Cameroun.

4. Ella, J. B. (2016). «Bayam-Sellam kyeossissiennes : logiques et stratégies des acteurs ». École Normale Supérieure, Université de Yaoundé I, Cameroun.

5. Folefack, D. P., Fongang, F. H. G., Mohamadou, A. K., Ebongue, J. P., Bikoi, A. \& Noupadja, P. (2017). Analysis of the plantain supply system of markets in the city of Douala. Journal of Economics and Sustainable Development. Vol 8, No. 6 (2017).

6. Folefack, D. P. \& Mohamadou, A. K., (2017). Approvisionnement en banane plantain des marches de la ville de Douala : contraintes et options stratégiques. Note politique CARBAP/IRAD/C2D/PAR PLANTAIN, N 3 mars 2017.

7. Fongang, F. G. H., Folefack, D. P., Pane, P. Z., Bokoï, A. \& Noupadja, P. (2016). Transformation et commercialisation des chips de banane plantain au Cameroun : une activité artisanale à forte valeur ajoutée. Int. J. Biol. Chem. Sci. 10 (3) : 1184-1198, June 2016.

8. Fouda, O. M. (2009). «Performance, financement et microcrédit dans les activités génératrices de revenus. Une étude empirique auprès des femmes Bayam-Sellam de Douala au Cameroun », in la vulnérabilité des TPE et des PME dans un environnement mondialisé, 11e Journées scientifiques du Réseau Entrepreneuriat, Trois-Rivières, Canada, INRPME.

9. Houngbo, E. (2014). Étude régionale sur la commercialisation de la banane plantain en Afrique de l'Ouest. CORAF/WECARD, Rapport final.

10. Ngo, N. W. M. (2011). Crise de l'État et stratégies de survie des femmes Bayam-Sellam au Cameroun. In Femmes, précarités, 
résilience. Les politiques sociales. $\mathrm{N}^{\circ} 1$ et 2 . Centre d'Études du Développement, Université Catholique de Louvain-la-Neuve (Belgique).

11. Mohamadou, A. K. (2016). Évaluation de la performance des circuits d'approvisionnement en banane plantain des marches de la ville de Douala. Mémoire de Master 2, Faculté d'Agronomie et des Sciences Agricoles (FASA), Université de Dschang.

12. Tomekpe, K. (2006). La banane plantain en Afrique, Fruit. Spécial issue 2006, p5.

13. Programme Alimentaire Mondial - PAM, (2013). Guide de l'analyse des marchés pour les programmes de transferts monétaires: Formulation, suivi et analyse d'impact. https://documents.wfp.org/stellent/groups/public/documents/manual_ guide_proced/wfp258666.pdf.

14. Pane, P. Z. (2015). Diagnostic socio-économique du système de production et de commercialisation des chips dans les régions du Littoral et du Centre au Cameroun. Mémoire de Master 2, Faculté d'Agronomie et des Sciences Agricoles (FASA), Université de Dschang.

15. Wandji, A.A. (2015). Dynamique des marchés transfrontaliers de la banane plantain de la vallée du Ntem (Sud Cameroun). Mémoire de Master II, ESSEC, Université de Douala. 103 p. 